\title{
Detection of Disrupters in the DC Protocol
}

\author{
Jurjen Bos \\ Bert den Boer \\ Centrum voor Wiskunde en Informatica, Amsterdam
}

\section{Introduction}

The Dining Cryptographers algorithm [Chaum 87] allows participants to send messages in a way ensuring that they cannot be traced.

Such sender anonymity, however, gives a new problem: participants can send so many messages that others may not send. We call this disruption. The interesting part of the problem is finding disrupters without reducing the untraceability of the other senders. A theoretical solution appears in [Chaum 87], but it is quadratic in the number of participants, and thus infeasible. Here, a efficient solution is presented that is practical.

\section{Overview}

We will first briefly introduce the known protocol. We then discuss several different kinds of disruption and efficient ways to prevent them. Finally, we introduce a new way to solve the collision problem without retries.

\section{The protocol}

The protocol is "unconditionally untraceable". This means that there is no mathematical way to determine the sender of a message, given the outputs of all participants. (There are ways to find the sender, of course, like asking everybody whether the message was theirs.)

Keys

We assume that the messages sent are, instead of field elements [Chaum 87], elements of an abelian group (written additively). This idea was proposed by 
[Pfitzmann 87]. The transmissions are divided into rounds. In each round a group element will be sent..

The protocol relies on the use of keys. A key is a uniformly distributed random group element that is known by two participants. A pre-arranged one of the participants will use the key, the other one will use the negative of the key. In the picture, we depict this with an arrow: the arrow points to the user who uses the positive value. This guarantees that the sum of all key values used is zero. Every participant then sends (effectively, broadcasts) the sum of her message with all the keys she shares. This is depicted with a gray oval. If she wants to send nothing, she should send zeroes (she broadcasts the sum of her keys only). The sum of the outputs of all participants-which can be computed from public information -is the sum of the messages of the participants.

We do not need to assume that every pair of participants shares keys. For details about the key sharing, see [Chaum 88].

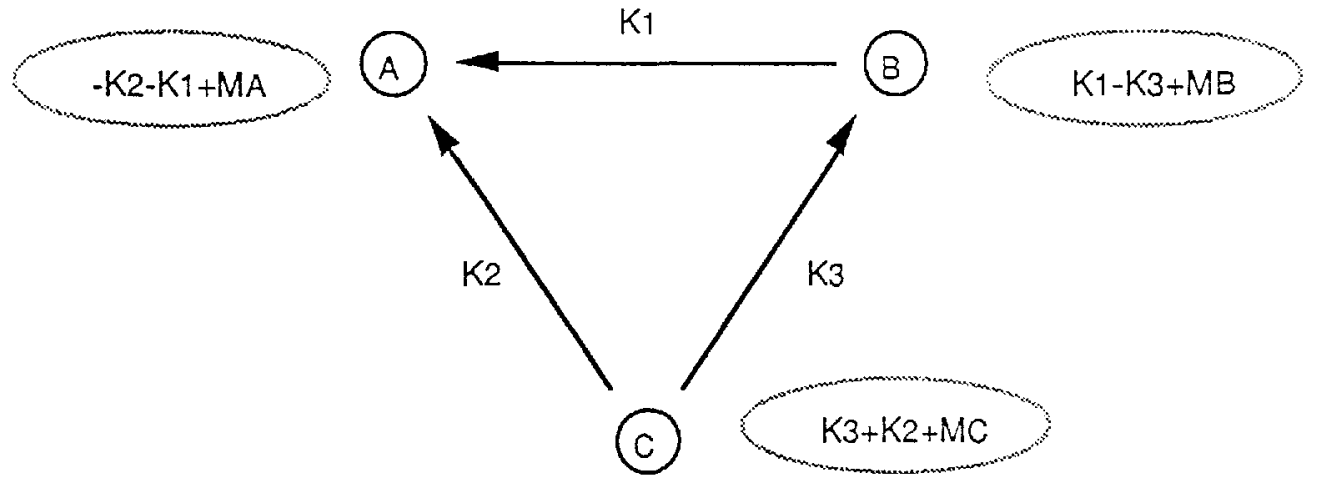

\section{Bus system}

The protocol can be viewed as a bus system. In this system, the output is accessible directly, while the input has to be coded. (This coding is the addition of the keys.)

Using this analogy, we can apply the current literature on bus systems. There are two differences between our bus system and those currently used: - If two participants send simultaneously (this is a collision), their outputs will be added. In current systems the result is just garbage. We will use this fact for making more advanced collision resolve schemes.

- It is impossible to trace a sender (this was a design goal). 
Bus systems need a protocol to assign the bus fairly to senders. We will call such a system a transmission rule. Current bus systems use the following properties for transmission rules:

- every user must be able to send a fair amount of data;

- the bus should be used as efficiently as possible.

In our problem, the rule needs one extra feature:

- the protocol may not limit sender anonymity.

\section{Disruption and Collisions}

Disruption is the sending of messages that disturb other messages. A collision is a number of messages that disturb each other, but are sent following to the transmission rule. A lot of current systems use collisions (for example, the ALOHA protocol).

In our system, things are more difficult, because the cause of a disruption or collision is difficult to find. To get a fair use of the channel, we seek a protocol that allows everybody to send, but restrict the amount of sending so that everybody has the same possibilities for using the channel. There are several ways to disrupt the system:

- Influencing the total directly. It is sometimes possible for a participant to influence the total regardless of what the others are sending, for example if someone can get hold of the last adder. In general, this will be easy to prevent and detect.

- Sending garbage. This can be the result of a faulty sender. This kind of disruption is easy to find.

- Sending too much. There does not exist a transmission rule that allows any participant to send as many messages as she likes. The amount that can be sent by a participant must be restricted in some way. Sending more messages than is allowed by the rule is also considered disruption.

\section{Opening}

Our only weapon in the fight against disrupters is opening. Opening a round of the protocol means that all information passed in this round must be made public. It is enough to only open the keys, because the other information is already public. It is only possible to open a round which does not contain any private information, so we need some trick to guarantee that there are rounds to open that do not compromise privacy, but that will catch a disrupter sending. 
The use of opening can be demonstrated by an example. We assume time is divided into periods, and that every sender is forbidden to send more than once in a period. We then have the following method that will sometimes catch a disrupter:

- If the number of messages in a period is greater than the number of participants, there is certainly a disrupter. He now can be caught.

- Open an anonymous voting protocol in which every participant claims a block. This reserved block is assumed to be her message and will not be opened for privacy reasons. Participants that did not send a block choose a random block to be reserved.

- At this point, there must be some unclaimed blocks left over. These can be opened without harming the privacy of non-disrupters. This will certainly yield a disrupter.

This system has the disadvantage that there can be collisions. The collisions are caused by the fact that senders send at a random moment (not to reveal their identity).

\section{Order of computation}

The total of the outputs from the participants can be computed in several ways. One of the first ways that comes to mind is to let the participants add up the total themselves, in a chain fashion:

- The first participant sends out her total.

- The later participants send out the sum of their total with the previous total.

- The output of the last participant is then the total to be broadcasted.

This method has the important drawback that the last participant completely determines the value of the total. She can use this fact to disrupt the protocol without being noticed.

Another way, which does not have this drawback, is a tree structure: The messages from two branches are added up using a stream adder to each new branch.

A second advantage of this system is that the turnaround time (the time it takes to compute the total from the bits of the participants) is only logarithmic in the number of participants.

\section{Transmission rules}

We now give an overview of several transmission rules. 


\section{Slot reservation}

This is the protocol originally proposed in [Chaum 88]. The idea is to fix in advance the time somebody is going to use. It works like this:

- There is a "reservation phase" that consists of a long stream of group elements. Everybody is obliged to send exactly one 1 value in this phase.

- If this phase does not contain as many l's as there are participants, there was a collision and the whole process is repeated again.

- The order of participants is the order in which they sent 1's.

This system has the disadvantage that participants can influence their time of sending, and that the overhead for the reservation phase is huge. The reservation phase has to have $200 \mathrm{n}^{2}$ bits to get a $99 \%$ probability of a collisionfree reservation phase.

\section{Collision detect}

It is a good idea to try to adjust the well-known ALOHA [Davies 79] system to our case: it works very well in practice in the case of a low average load. The idea is that a participant sends whenever she has something to send. If the average load is low, this will not cause a collision in most cases, so there is no delay. If there is a collision, she must try again after some time. If we limit the amount of sending, we can catch disrupters with opening, as we showed before. In this case, collisions cause a very long delay, because of the limitation on the sending.

\section{The collision resolve algorithm}

The additive property of the system gives us the opportunity to use the result of a collision. By prepending each message with a header, it is possible to find out the order in which the participants have to send without any further messages.

Like in the slot reservation protocol, the size of the field must be sufficient to give a reasonable probability that the header messages are different. (For example, to have a probability of $99 \%$ that ten different headers are different, we need at least 4481 field elements).

We let participant $i$ send as header the message $R_{i}, A_{i}, A_{i}^{2}, A_{i}^{3}, \ldots, A_{i}^{k}$. Assume now that $n$ participants collide, so that the added-up result of those $n$ 
simultaneous headers is $\sum_{i=1}^{n} R_{i}, \sum_{i=1}^{n} A_{i}, \sum_{i=1}^{n} A_{i}^{2}, \sum_{i=1}^{n} A_{i}^{3}, \ldots, \sum_{i=1}^{n} A_{i}^{k}$. Here we assume (without loss of generality) that the participants are numbered 1 to $\mathrm{n}$. We will write the sums of the $A_{i}^{j}$ as $S_{1}, S_{2}, \ldots, S_{k}$.

We now need an algorithm that assigns each of the $n$ colliding participants a unique number in the range $1 \ldots n$. We will construct the algorithm from a standard technique [Lidl 71] to find the $A_{i}$.

Observe the polynomial $P(x)=\prod_{i=1}^{n}\left(1-x A_{i}\right)$, which has as roots the reciprocals of the $A_{i}$. We can write this out as $\sum_{j=0}^{n} \sigma_{j} x^{j}$, with

$$
\begin{aligned}
& \sigma_{0}=1 \\
& \sigma_{1}=-\sum_{i=1}^{n} A_{i}, \\
& \sigma_{2}=\sum_{i=1}^{n-1} \sum_{i^{\prime}=i+1}^{n} A_{i} A_{i^{\prime}}, \text { and so on. }
\end{aligned}
$$

We can rewrite these equations as $\left({ }^{*}\right)$

$S_{1}-\sigma_{1}=0$,

$S_{2}-\sigma_{1} S_{1}+2 \sigma_{2}=0$, up to

$S_{n}-\sigma_{1} S_{n-1}+\ldots+(-1)^{n-1} \sigma_{n-1} S_{1}+(-1)^{n} n \sigma_{n}=0$.

In this case, the equations are easy to solve, because it is a linear triangular system. We will see later on that in the binary case it is different. Having the coefficients of the polynomial $P$, we can find its zeroes. Because $P$ completely factors in monomials, and $P(0) \neq 0$, we can use that $P(x) \mid x^{p-1}-1$. Because $x^{p-1}-1=\left(x^{\frac{p-1}{2}}+1\right)\left(x^{\frac{p-1}{2}}-1\right)$, we have $P(x)=\operatorname{gcd}\left(P, x^{\frac{p-1}{2}}+1\right) \cdot \operatorname{gcd}\left(P, x^{\frac{p-1}{2}}-1\right)$. We will call $P_{1}^{\prime}(x)=\operatorname{gcd}\left(P, x^{\frac{p-1}{2}}+1\right)$ and $P_{2}^{\prime}(x)=\operatorname{gcd}\left(P, x^{\frac{p-1}{2}}-1\right)$.

To now define an order on the factors of $P$, we define one of the two factors as the "earlier" factor, and the other as the "last" factor. Later on we will define a way to do this. If a participant wants to know the place of her value $A_{i}$, she can quickly find out in which of the two parts her factor $x-A_{i}$ occurs by 
finding out whether $P_{1}^{\prime}\left(A_{i}\right)$ or $P_{2}^{\prime}\left(A_{i}\right)$ is zero. If her factor occurs in the factor which is defined as the "last" factor, then the order number is the degree of the "first" factor plus the order number in the "last" factor. This means that further investingation of the "first" factor is not necessary. Repeating the process on the factor that contains $x-A_{i}$ yields the order number of the $A_{i}$ in expected ${ }^{2} \log n$ steps. We can repeat the process using the factorizations $x^{p}-x=\left((x+i t)^{\frac{p-1}{2}}+1\right)\left((x+i t)^{\frac{p-1}{2}}-1\right)(x+i t)$ for a prearranged $t$, and $i$ increasing from 1 upwards.

We now have an algorithm that allows a participant to find the place of her $A_{i}$, but is is yet predictable: a sender can (approximately) influence the resulting order by choosing another value. We can solve this by letting each participant send another random value $R$ in the header (so that the header looks like $R, A_{i}, A_{i}^{2}, A_{i}^{3}, \ldots, A_{i}^{k}$ ), and using the sum of those $R$ 's determine the resulting order. For example, one can use the bits of the sum of the $R$ to determine whether $P_{1}^{\prime}\left(A_{i}\right)$ or $P_{2}^{\prime}\left(A_{i}\right)$ is defined to be the "earlier" factor.and also take $t$ to be the sum of the $R$ (assuming this sum is not zero).

The polynomials we use do not guarantee that the process will end quickly; if this is a problem, we can define an "escape rule" for those $A_{i}$ that do not get separated after a prearranged number of steps. This means that participant who factor out their monomial in this number of steps will not notice the problem. The participants who need extra steps can use random choices to factor the remaing polynomial, and order the subset of the $A_{i}$ just in normal increasing order. If necessary, we can then use another rule for making the order unpredictable.

\section{The binary case}

We now assume we have a field of $2^{r}$ elements. In this case, the values of $S_{j}$ for even $j$ are useless (because $S_{2 j}=S_{j}^{2}$ ). This means that we have to send $A_{i}, A_{i}^{3}, A_{i}^{5}, \ldots, A_{i}^{2 k-1}$ to have enough equations in (*). The resulting system for the $\sigma_{i}$ is now harder to solve; a nice solution occurs in [Burton 71]. Factoring the polynomial is also a little bit different.

We factor the polynomial $x^{2^{r}}-x$ into $G(x)(G(x)+1)$ where $\mathrm{G}$ is the absolute trace function [Lidl, page 170]:

$$
G(x)=\sum_{i=0}^{r-1} x^{2^{i}}=x+x^{2}+x^{4}+\ldots+x^{2^{r-1}} \text {. Using this factorization and }
$$


succesively the factorizations $G\left(y^{i} x\right)\left(G\left(y^{i} x\right)+1\right)$ of the polynomial $x^{2^{r}}-x$ for $i$ in $1, \ldots, r-1$ (where $1, y, \dot{y}^{2}, \ldots, y^{r-1}$ is a basis for $G F\left(2^{r}\right)$ ), we can apply the same method as above to find the position of the $A_{i}$. An advantage is that the factorization will end in a relatively short time, so that an escape rule is not necessary.

\section{References}

[Burton 71] Herbert O. Burton: "Inversionless Decoding of Binary BCH Codes", IEEE transactions on Information Theory, Vol. IT-17, No. 4, July 1971.

[Chaum 87] D. Chaum: "The Dining Cryptographers Problem: Unconditional sender and Recipient Untraceability". Journal of Cryptology (1988) 1:65-75.

[Davies 79] D. W. Davies, D. L. A. Barber, W. L. Price, C. M. Solomides: "Computer Networks and their Protocols", John Wiley and Sons, 1979.

[Lidl 83] Rudold Lidl, Harald Niederreiter: "Finite fields","Encyclopedia of mathematics and its applications", Vol. 20, section Algebra.

[Pfitzmann 87] A. Pfitzmann: "Dienseintegrierende Kommunaktionsnetze mit Teilnehmer-überprüfbarem Datenschutz", Ph. D. thesis, 1987. 